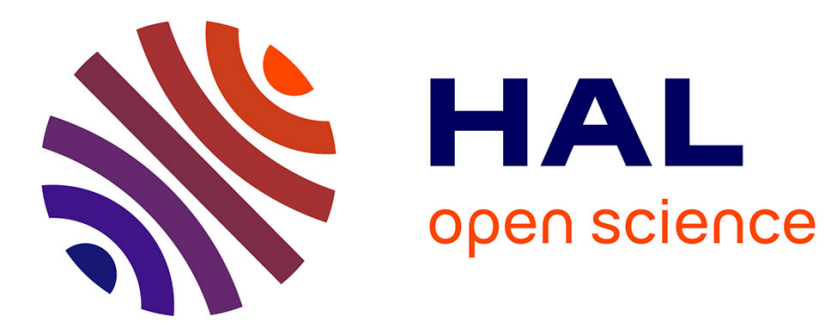

\title{
directPath: An Expert Path Planning Framework to Handle Environment Knowledge
}

\author{
Romain Jacob, Karl J Hedrick
}

\section{To cite this version:}

Romain Jacob, Karl J Hedrick. directPath: An Expert Path Planning Framework to Handle Environment Knowledge. IEEE CYBER 2014, 4th Annual International Conference on Cyber Technology in Automation, Control, and Intelligent Systems, Jun 2014, Hong-Kong, China. pp.7-12, 10.1109/CYBER.2014.6917427 . hal-01274951

\section{HAL Id: hal-01274951 \\ https://hal.science/hal-01274951}

Submitted on 16 Feb 2016

HAL is a multi-disciplinary open access archive for the deposit and dissemination of scientific research documents, whether they are published or not. The documents may come from teaching and research institutions in France or abroad, or from public or private research centers.
L'archive ouverte pluridisciplinaire HAL, est destinée au dépôt et à la diffusion de documents scientifiques de niveau recherche, publiés ou non, émanant des établissements d'enseignement et de recherche français ou étrangers, des laboratoires publics ou privés. 


\section{directP ath : An Expert Path Planning Framework to Handle Environment Knowledge}

CONFERENCE PAPER · JUNE 2014

DOI: 10.1109/CYBER.2014.6917427

READS

14

2 AUTHORS, INCLUDING:

Romain Jacob

ETH Zurich

2 PUBLICATIONS 2 CITATIONS

SEE PROFILE 


\section{directPath : An Expert Path Planning Framework to Handle Environment Knowledge}

\author{
Romain JACOB \\ Vehicle Dynamics and Control Lab \\ University of California BERKELEY, CA, USA \\ From : École Normale SuprieURe de Cachan, FRANCE \\ Email: rjacob@ens-cachan.fr
}

\author{
J. Karl HedRICK \\ Professor of Mechanical Engineering \\ University of California BERKELEY, CA, USA \\ Email : khedrick@me.berkeley.edu
}

\begin{abstract}
The main purpose of this paper is to present a new path planning framework, called directPath, aimed to handle a priori environment knowledge efficiently. Our algorithm is an expert system designed to specifically exploit that knowledge, and then eventually collaborate with other frameworks in order to improve overall path planning performances.

It performs a forward search with partial heuristic. It is iterative and can return a partial but safe path when terminated early. Moreover, unlike classical search algorithms, its design allows a quick detection of unfeasible problems.

In this paper, we present the framework structure and illustrate its performances and properties by simulations. We will show that directPath can complete the planning task very quickly, with only minor optimality loss.
\end{abstract}

\section{INTRODUCTION}

Since the 1980s, a tremendous amount of work has been done on real-time path planning for autonomous mobile robots. The classic formulation for a path planning problem is the following : Given an initial position and orientation of an agent and a goal position, a path planner will generate a continuous free path starting at the initial position and orientation terminating at the goal position, if such path exists, and report a failure otherwise [1].

Two main approaches are used to deal with this classic path planning problem. There are referred to as global and local approaches.

Global approaches use a mapping of the entire accessible environment to solve the path planning problem. That insures the property of global convergence. The path planning problem will be solved, if feasible. However, building and eventually updating a global map is a heavy computational burden to bear for a robot. That is one of the main challenges for Simultaneous Localization And Mapping (SLAM), which is currently heavily studied [2]-[4].

Local sensor-based path planning frameworks, on the other hand, use latest sensor data to plan future actions in a reactive/adaptive way. Most commonly used approaches include potential field methods (also known as virtual forces [5] [6]), behavior-based systems [7] [8] and fuzzy logic approaches [9] [10].

Although they are usually much simpler to implement than global ones, local planners may get trapped in a local minimum and subsequently follow a diverging path or a loop, while attempting to escape from it. Thus, it is not realistic to rely only on local planners. As a result, researchers are commonly combining different approaches to improve overall performances [11].

Similarly, the directPath algorithm we present here can be coupled with classical frameworks. Indeed, these aim to be useful in real application cases, where we often have only partial or no prior knowledge of the environment. As a result, it led us toward planning frameworks which do not benefit from such a priori knowledge when there is some, or at least not efficiently.

Our algorithm is an expert system designed to specifically exploit that knowledge, and then eventually collaborate with other frameworks to optimize overall performances. It aims to quickly detect unfeasible problems and to reduce computation time.

\section{Problem FORMUlation}

We are considering a standard path planning problem in a known environment. In $\mathbb{R}^{2}$, given a starting point $S$, a goal point $G$, and a set of obstacles $\mathbb{O}$, find the minimum length path $\mathbb{P}$, string of points that connects $S$ and $G$ without intersecting with any obstacle. Obstacles are closed polygons. They can be either convex or non-convex.

To solve this problem, we present a new framework, called directPath, which efficiently handles a priori environment knowledge. It is able to complete the path planning task on its own, or can be used as a support to other frameworks, in a hybrid manner.

The main notations and functions used in directPath are listed below :

$s \quad$ Current starting point, for a given iteration ;

$g \quad$ Current goal point, for a given iteration ;

$G_{(c) c w}$ Subgoal defined by (counter)clockwise wall-

following - Refer to Section III-A2 ;

$\overrightarrow{A B} \quad$ Straight-line segment from point $A$ to point $B$;

$\overrightarrow{A B} \quad$ Length of segment $\overrightarrow{A B}$;

$\mathbb{P}=\left\{P_{1}, \ldots, P_{n}\right\} \quad$ Path, string of points, vertices of the path from $P_{1}$ to $P_{n}$, composed of straight-lines segments $\overrightarrow{P_{i} P_{i+1}}$;

$\mathbb{O}=\left\{O_{1}, \ldots, O_{m}\right\} \quad$ Set of obstacles in the environment ; 


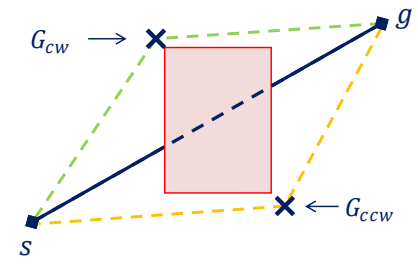

Figure 1. Only two ways to go around an obstacle.

getIntersection $(A, B, \mathbb{O}) \quad$ Returns a pair $\mathbb{I}=(I, O)$, where - $I$ is the closest of the intersection points between $\overrightarrow{A B}$ and $\mathbb{O}$ from point $A$,

- $O$ is the associated obstacle, if any.

Returns $\emptyset$ otherwise ;

defineSubGoals $(s, g, \mathbb{I}) \quad$ Returns the set of subgoal options: $\operatorname{SuB}_{g}=\left\{G_{c w} ; G_{c c w}\right\}$; chooseSubGoal $\left(s, g, \mathrm{SuB}_{g}\right)$ Returns chosen subgoal : $s u b_{g}$; update $\left(s, g, \mathbb{P}, \mathbb{I}, s u b_{g}\right) \quad$ Returns $s$ and $g$ for next iteration and extends the current path $\mathbb{P}$; closestNeigh $(A, O) \quad$ Returns the closest safe position wallFollow $(A, O$, dir $)$ around obstacle $O$ from point $A$; Returns next boundary position of obstacle $O$, from point $A$. Direction dir can be clockwise (cw) or counterclockwise (ccw) ; $\operatorname{is} \operatorname{Valid}(A)$ Returns 1 if $A$ is a walkable state. Returns 0 otherwise.

\section{FRAMEWORK DESCRIPTION}

This paper aims to present a path planning framework which efficiently benefits from environment knowledge and that can quickly detect unfeasible problems.

We will discuss that directPath can either solve the planning task on its own (Section IV) or be coupled with other path planning frameworks, in a hybrid manner, to improve overall path planning performances (Section V).

\section{A. Framework structure}

The directPath framework is built on a simple fact. When one wants to go in a direction and encounters an obstacle, there are only two options. It can go around the obstacle clockwise, or counterclockwise. As illustrated in Figure 1, up to two subgoals can be found, from which the obstacle is no longer on the way. Ultimately, the path planning task can be reduced to a succession of choices between subgoal options.

directPath uses that idea to produce a valid path. First, it tries to go straight to the current goal, $g$. For any obstacle on its way, subgoal options are generated and a choice is made. The chosen subgoal becomes the new current goal to reach. The previous one is saved and will be retrieved once the subgoal will be reached. The algorithm iterates until the initial goal, $G$, is reached. Algorithm 1 is directPath pseudo-code.

In the following, we describe in more details the main functions called in directPath.

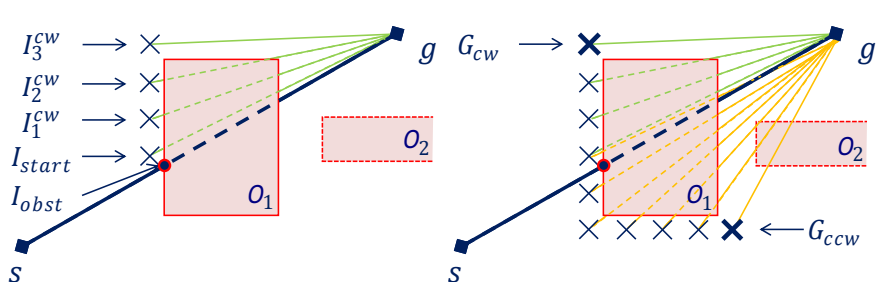

(a)

(b)

Figure 2. Subgoals definition method : (a) wallFollow $\left(I_{\text {start }}, O_{1}, c w\right)$; (b) $\operatorname{SuB}_{g}=\left\{G_{c w} ; G_{c c w}\right\}$.

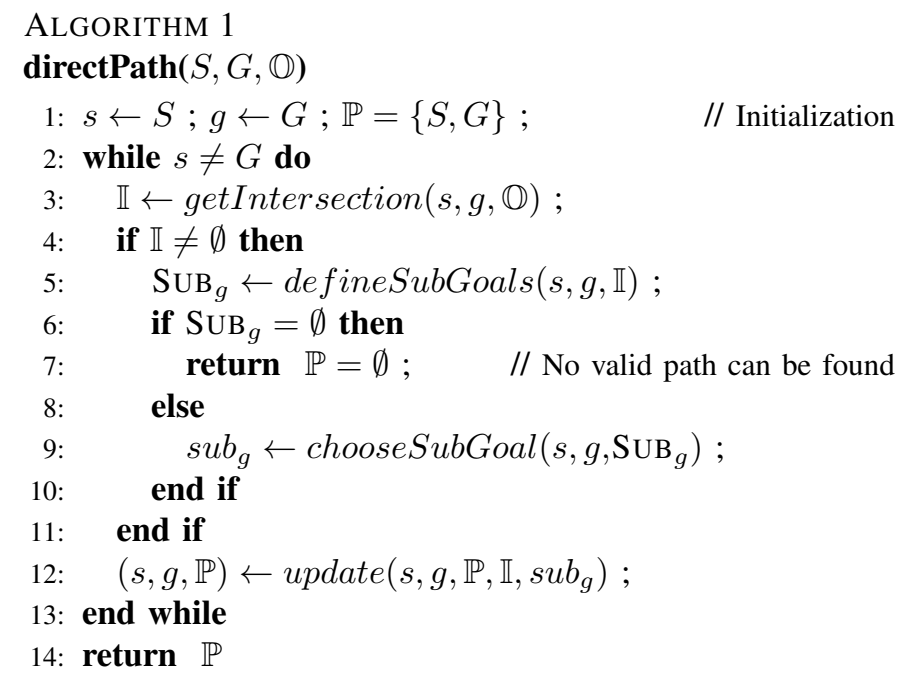

1) getIntersection $(s, g, \mathbb{O})$ : This function takes two points $s$ and $g$, and a set of obstacles $\mathbb{O}$ as inputs. It checks whether or not $\overrightarrow{s g}$ intersects with any obstacles of $\mathbb{O}$. It returns a pair $\mathbb{I}=\left(I_{\text {obst }}, O_{\text {ind }}\right)$, where $I_{\text {obst }}$ is the closest intersection point from $s$ (as illustrated in Figure 2(a)). $O_{\text {ind }}$ is the obstacle on the way. If no obstacle lies between $s$ and $g$, getIntersection () returns $\mathbb{I}=\emptyset$.

2) defineSubGoals $(s, g, \mathbb{I})$ : This function takes $s, g$ and $\mathbb{I}$ as inputs and returns the set of subgoal options : $\mathrm{SUB}_{g}=$ $\left\{G_{c w} ; G_{c c w}\right\}$. Details are shown in Algorithm 2.

Notice that the function takes $\mathbb{I}$ (and not $\mathbb{O}$ ) as input. It implies that only $O_{i n d}$ (the first obstacle on the way) is considered while defining subgoals (e.g., in Figure 2 case, obstacle $\mathrm{O}_{2}$ is ignored). It means that once one reaches a subgoal, it will be able to go straight from it to $g$ if no other obstacle is on the rest of the way. If there are, the algorithm will iterate to reach $g$, and ultimately to reach the initial goal, $G$. Refer to Algorithm 1.

In some cases, the wallFollow() function might fail to find a valid subgoal. For instance, if wallFollow() takes the agent outside of the environment boundaries, or in a nonaccessible location (e.g., a too narrow corridor). This is how the algorithm detects unfeasible problems. If both clockwise and counterclockwise wall-followings fail, directPath will return an empty path : the obstacle on the way is unpassable. Refer to lines 5-7 in ALGORITHM 1. 


\section{ALGORITHM 2}

defineSubGoals $(s, g, \mathbb{I})$

1: $I_{\text {start }} \leftarrow$ closestNeigh $\left(I_{\text {obst }}, O_{\text {ind }}\right)$;

2: $I_{0}^{c w} \leftarrow I_{\text {start }} ; k \leftarrow 0 ; \quad$ // cw wall-following

3: repeat

4: $\quad I_{k+1}^{c w} \leftarrow$ wallFollow $\left(I_{k}^{c w}, O_{\text {ind }}, c w\right) ; k \leftarrow k+1$;

5: until $\left(\operatorname{get}\right.$ Intersection $\left(I_{k}^{c w}, g, \mathbb{O}\right)=\emptyset$

or $\left./ i \operatorname{Valid}\left(I_{k}^{c w}\right)\right)$

6: if $i s \operatorname{Valid}\left(I_{k}^{c w}\right)$ then

7: $\quad G_{c w} \leftarrow I_{k}^{c w}$;

8: else

9: $\quad G_{c w}$ is undefined ;

10: end if

11: $I_{0}^{c c w} \leftarrow I_{\text {start }} ; k \leftarrow 0$;

12: repeat

13: $\quad I_{k+1}^{c c w} \leftarrow$ wallFollow $\left(I_{k}^{c c w}, O_{\text {ind }}, c w\right) ; k \leftarrow k+1$;

14: until ( getIntersection $\left(I_{k}^{c c w}, g, \mathbb{O}\right)=\emptyset$ or $\left./ i s \operatorname{Valid}\left(I_{k}^{c c w}\right)\right)$

15: if $i s \operatorname{Valid}\left(I_{k}^{c c w}\right)$ then

16: $\quad G_{c c w} \leftarrow I_{k}^{c c w}$;

17: else

18: $G_{c c w}$ is undefined ;

19: end if

20: return $\mathrm{SuB}_{g}=\left\{G_{c w} ; G_{c c w}\right\}$

3) chooseSubGoal $\left(s, g, \mathrm{SUB}_{g}\right)$ : This function takes $s, g$ and the set $\mathrm{SUB}_{g}$ as inputs and returns the next subgoal to go to : $s u b_{g}$. When both $G_{c w}$ and $G_{c c w}$ have been successfully defined, we need to make a choice : Which subgoal should we try to reach before heading again to $g$ ?

Depending on the situation, this is performed either by the use of a heuristic or by considering previous choices made. For every obstacle, the first subgoal is always picked by heuristic(). If more than one subgoal is needed to get pass the same obstacle, then history () is used. Both sub-fuctions are described below :

a) heuristic $\left(s, g, \mathrm{SUB}_{g}\right)$ : This function takes $s, g$ and $\mathrm{SUB}_{g}$ as inputs and returns a pair of path lengths $\mathbb{H}=\left(H_{c w} ; H_{c c w}\right)$. These lengths are estimates of the euclidean length of paths $\left\{s, G_{(c) c w}, g\right\}$, which we want to minimize. They are computed as follows :

$$
H_{(c) c w}=l_{1}^{(c) c w}+d^{(c) c w}+l_{2}^{(c) c w}
$$

Where $l_{1}^{(c) c w}=\overline{s G_{(c) c w}}$ and $l_{2}^{(c) c w}=\overline{G_{(c) c w} g} \cdot d^{(c) c w}$ is presented in the next paragraph.

It turns out that $l_{1}^{(c) c w}$ is not sufficient to provide a good estimate of the final length of $\left\{s, G_{(c) c w}\right\}$. As illustrated in Figure 3, it does not take into account that we have to go around the obstacle to reach $G_{(c) c w} . d^{(c) c w}$ is added to the heuristic to solve that problem. It is defined as the maximal distance we get away from $\overrightarrow{s g}$ during the wall-followings performed in defineSubGoals(). It is an estimate of the

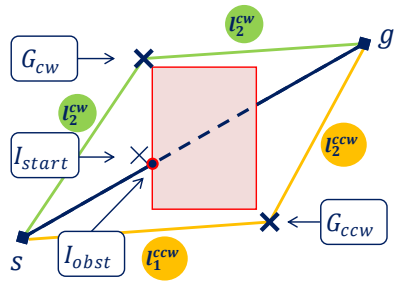

(a)

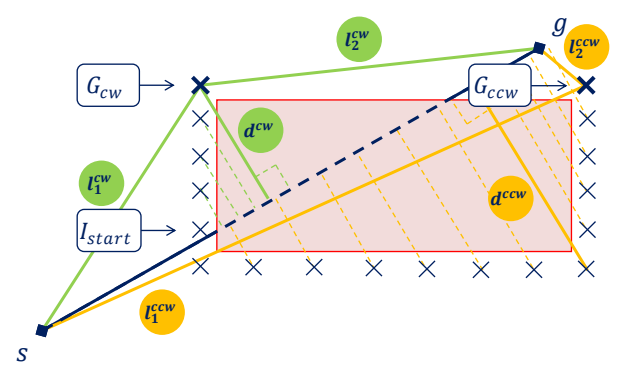

(b)

Figure 3. Heuristic definition : (a) Definition of $l_{1}^{(c) c w}$ and $l_{2}^{(c) c w}$; (b) Definition of $d^{(c) c w}$.

penalty for having to go around the obstacle, instead of going straight to $G_{(c) c w}$. This is illustrated in Figure 3(b).

Simulations have shown this heuristic to be sufficient to pick the best subgoal.

b) history () : This function takes previous choices made as inputs and returns $s u b_{g}$. When several subgoals are needed to get pass the same obstacle, the algorithm makes choices consistent with the previous ones.

For example, consider Figure 3(b) case. If we were to choose $G_{c c w}$ (which is not optimal since $H_{c w}<H_{c c w}$ ), we would need to pick another subgoal afterwards to reach it. Then directPath would keep getting pass the obstacle counterclockwise, to provide consistency to the choosing strategy. This avoids to get trapped in a local minimum, which, in our case, would be a loop, a cyclic choices of the same subgoals.

In a maze, one will ultimately get out by always turning in the same direction. Similarly, our strategy assure to get pass any obstacle, if it can be.

Consequences of this approach in term of completeness and optimality will be further discussed in CONCLUSIONS (Section VI).

4) update $\left(s, g, \mathbb{P}, \mathbb{I}, s u b_{g}\right)$ : This function takes $s, g, \mathbb{P}, \mathbb{I}$ and $s u b_{g}$ as inputs. It returns $s$ and $g$ for the next iteration (while loop line 2 of ALGORITHM 1). Eventually, it extends the current path $\mathbb{P}$.

Let us assume the current path is :

$$
\mathbb{P}=\{S, w p_{1}, \ldots \overbrace{w p_{k}}^{s}, \overbrace{w p_{k+1}}^{g}, w p_{k+2}, \ldots, G\}
$$

a) If $\mathbb{I}=\emptyset$ : No obstacle lies between $s$ and $g$. We only have to increment current starting point $s$ and goal point $g$.

$$
\left\{\begin{array}{lll}
s & \longleftarrow & w p_{k+1} \\
g & \longleftarrow p_{k+2}
\end{array}\right.
$$




$$
\Rightarrow \mathbb{P}=\{S, w p_{1}, \ldots w p_{k}, \overbrace{w p_{k+1}}^{s}, \overbrace{w p_{k+2}}^{g}, \ldots, G\}
$$

b) If $\mathbb{I} \neq \emptyset$ : An obstacle is on the way and a subgoal $s u b_{g} \in\left\{G_{c w} ; G_{c c w}\right\}$ has been returned by chooseSubGoal(). In that case, the current starting point $s$ remains, $s u b_{g}$ is set as new current goal point and the path is extended.

$$
\begin{aligned}
& \left\{\begin{array}{lll}
s \longleftarrow & \longleftarrow p_{k} \\
g & \longleftarrow & \longleftarrow b_{g}
\end{array}\right. \\
& \Rightarrow \mathbb{P}=\{S, w p_{1}, \ldots \overbrace{w p_{k}}^{s}, \overbrace{s u b_{g}}^{g}, w p_{k+1}, w p_{k+2}, \ldots, G\}
\end{aligned}
$$

\section{B. Overview of framework properties}

In the last section, we described the main functions called in directPath. Here, we highlight the main properties of that framework.

Most importantly, if the planning task is unfeasible, directPath terminates as soon as the unpassable element (e.g., an absolute barrier or a too narrow corridor) is encountered. If defineSubGoals() returns an empty set, the algorithm stops and returns an empty path without wasting any more time.

Moreover, the algorithm plans in a forward manner. Once first segments of the path are returned, they will be on the final path. Then, even if the rest of the path is computing, the agent can start tracking the beginning of the path, without any efficiency loss.

Finally, since it is completely built on geometry, directPath is deterministic. This avoids problems that might occur with biased random methods, in which randomness sometimes produces unexpected long computation times.

In the next section, we present an empirical evaluation of some properties and performances of directPath.

\section{EMPIRICAL EVALUATION}

Simulations have been performed to illustrate and evaluate two key performances of the framework : computation speed and path efficiency. We used a basic scenario, described in the following, to compare directPath with a standard $A^{\star}$ framework [12].

\section{A. Simulation scenario}

Given a 2 dimensional squared grid world (later referred to as a field) and set of obstacles $\mathbb{O}$, an agent has to find its way between a starting point $S$ and goal point and $G$. The field is a room-like environment. The agent cannot go out of the boundaries. Allowed actions are cardinal and diagonal moves.

In this scenario, agent shape is not considered : it is limited to a simple dot, which gives a 2 dimensional problem (X and $\mathrm{Y}$ coordinates, the agent orientation can be omitted due to that assumption). This model is sufficient since obstacle boundaries can be arbitrary expended to compensate for an agent of nonzero size [13]. Moreover, the dimensional loss doesn't matter since we mainly want to compare performances of directPath and $A^{\star}$.

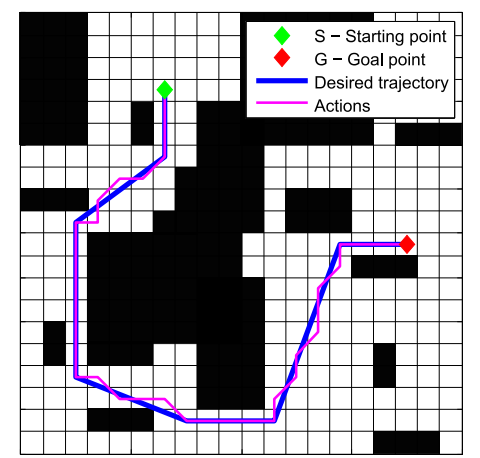

Figure 4. Example of scenario : $N=250$ and $h_{v a l} \approx 0.35$

Among others, we focus on two important parameters : the number of walkable states in the field, $N$ (the size of the state space), and the heuristic value of a scenario, later referred to as $h_{v a l}$. It is defined as the ratio of the euclidean distance and the length of the shortest path between $S$ and $G$ :

$$
h_{v a l}=\frac{\overline{S G}}{\text { length of the optimal path }}
$$

Therefore, the smaller the $h_{v a l}$, the harder the scenario is to solve. If $h_{v a l}=1$, there is no obstacle between $S$ and $G$.

Fields were extracted from an open repository of room maps, mazes and random maps [14]. From 150 different maps, we kept parts of different size (e.g., 20 by 20, 100 by 100 , ...) to get a wide range of $N$ values. $S$ and $G$ were picked randomly. Path lengths are measured with diagonal moves having cost $\sqrt{2}$ and cardinal ones having cost 1 .

To ease the results analysis, we regrouped data points into buckets. Each bucket represents an interval of $N$ and $h_{v a l}$ (e.g., $1.10^{4}<N<5.10^{5}$ and $0.4<h_{\text {val }}<0.5$ ) and contains at least 10 data points.

\section{B. Framework evaluation}

We evaluate directPath performances through two key features : the average computation time to solve the path planning problem, and the efficiency of the returned path, later referred to as $\eta$. Simulation results are allocated into $N$ and $h_{v a l}$ buckets. Figures show mean values over the buckets.

Our framework is compared with a basic $A^{\star}$ algorithm, which uses euclidean distance to the goal as heuristic, no tie-breaking rule and no embedded way of using a priori information about obstacles shape and location.

Simulations were run with an Intel Core 17-3740QM, 2.70GHz CPU and 8GB RAM.

1) Computation time : Computation time results are presented in Figure 5.

The blue lines, associated to the left-hand Y-axis, represent the mean computation time for directPath to solve the problem. Red bars represent standard deviation over each bucket. The green doted-lines, associated to the right-hand Yaxis, represent the time ratio between directPath and $A^{\star}$. 

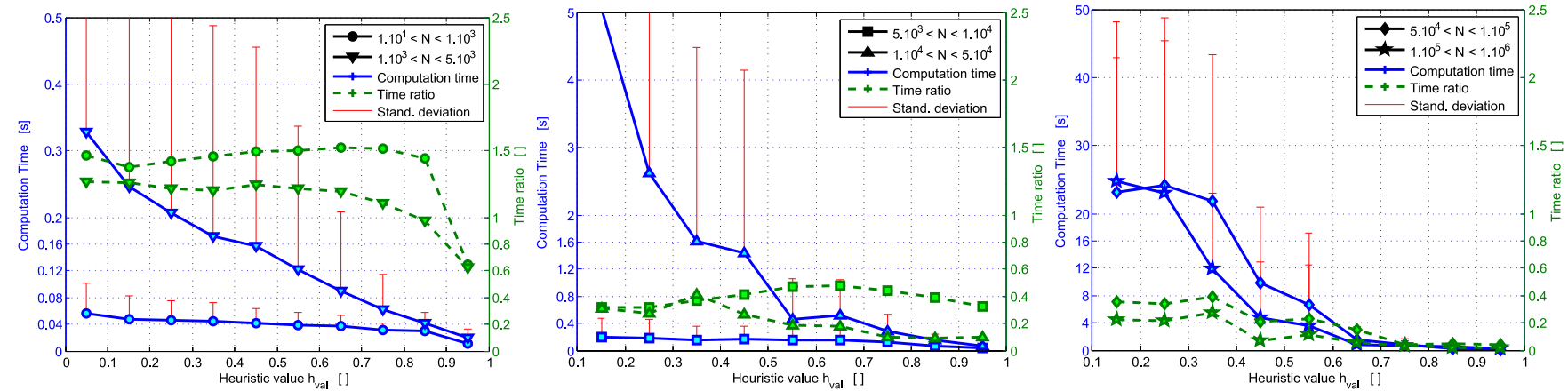

Figure 5. Computation time for different $h_{v a l}$ and six classes of state space size $N$

When the ratio is smaller than one, directPath is faster than $A^{\star}$.

From these results, we can say that directPath completes fairy quickly. For instance, it takes no more than 0.4 second to find a path in fields of 10000 states, even for the most difficult scenario (when $h_{v a l}$ is very small).

Standard deviation is higher for difficult scenario. This is mainly because we do not consider the optimal path length of a scenario while allocating it in a bucket. In most difficult scenario, path lengths can vary a lot, and so the computation time. Optimal path length would be a pertinent third parameter for further performances evaluation.

Naturally, both algorithms require more time when difficulty increases (when $N$ grows and $h_{v a l}$ drops). However, time ratio illustrates how the computation time of both algorithms evolves with scenario challenge. It shows that directPath performs better, compared to $A^{\star}$, when $N$ grows (up to 20 times faster when $N>10^{5}$ ) ; but this benefit diminishes with $h_{v a l}$.

2) Path efficiency $\eta$ : Another key feature of a path planning framework is the optimality of the paths it returns with respect to a given cost function. In our case, we want to minimize the total length of the path. We define the path efficiency $\eta$ as :

$$
\eta=\frac{\text { length of the optimal path }}{\text { length of returned path }}
$$

The optimal path length is obtained by using $A^{\star}$, which is proven to be optimal [12]. So $\eta$ provides a good metrix for optimality.

Simulation results are presented in Figure 6.

As expected, the efficiency of directPath decreases with the problem challenge. We mentioned in Section III that suboptimality mainly comes from the decision making process. So naturally, the more decisions directPath makes, the more likely it is to return a suboptimal path.

However, Figure 6 shows a fairly small loss of optimality (most of the time below 5\%). The average efficiency of returned paths remains close to 1 , which correspond to the optimal path returned by the $A^{\star}$ algorithm.

We can also note that $\eta$ seems higher when $h_{v a l}$ is very small. This is because smaller $h_{v a l}$ scenario mainly come from

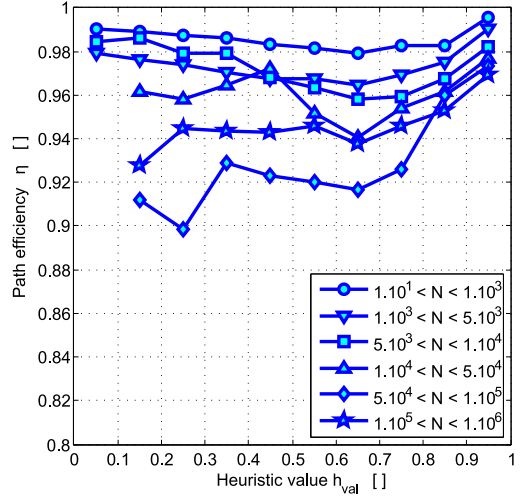

Figure 6. Path efficiency $\eta$ for different $h_{v a l}$ and six classes of state space size $N$

mazes, in which there are in general only few options to reach the goal. Ultimately, if there were only one way to get to the goal, directPath would necessarily return the optimal path, since no suboptimal decisions can lead to the goal.

\section{POSSIBle HYBRID USE OF directPath}

Simulations presented in the last section allow us to say that directPath can provide valid paths quickly, with only small optimality loss. In this section, we point out some possible benefit for a hybrid use of directPath with other classical frameworks.

\section{A. Quick detection of unfeasible problems}

As presented in Section III, a key property of directPath design is its ability to quickly detect unfeasible problems.

This can be very useful when the state space size grows. Indeed, for search algorithms like $A^{\star}$, time to detect unfeasibility is at least of the order of the number of reachable states from the starting point. Which can be huge.

\section{B. Hybrid use with $A^{\star}$}

Consider using directPath to obtain a succession of subgoals from $S$ to $G$. Then, instead of asking $A^{\star}$ to produce the complete path, subdivide the planning task, asking for a path to the first subgoal, and iterate until the initial goal $G$ is reached. 
This is to say that we use a new (inconsistent) heuristic in a $A^{\star}$ framework. It would be the euclidean distance to next subgoal. It can also be considered as a scale reduction approach.

That would allow to deal with higher dimensional problems. For instance directPath would return directions for $\mathrm{X}$ and Y coordinates of the agent while $A^{\star}$ would handle its shape and orientation. However, it would inherit the suboptimality of directPath.

\section{Hybrid use with a potential field approach}

Consider using the original path returned by directPath as a guide to avoid local minimum in a potential field framework.

That could be very interesting in applications with complex motion constrains (e.g., kinematics plus dynamics) and in dynamic environments, both aspects well-handled by potential field approaches.

These claims are no proof for a general benefit of using directPath in hybrid frameworks. However, it seems fair to say that further studies on this subject are worth being considered.

\section{Conclusions}

directPath aims to efficiently benefit from a priori environment knowledge. Therefore, speed is a key feature of the algorithm. We showed in Section IV that the path planning task is completed fairly quickly. Moreover, directPath can quickly detect unfeasible problems. The order of directPath completion time is $O(n \times l)$, where $n$ is the number of obstacles and $l$ the order of their perimeter. This has not be discussed here because of space limitation. Eventually, this would make it suitable for on-line planning.

However, it returns suboptimal paths. As presented in Section III, this comes from both the subgoal definition method (defineSubGoals()) and the choosing strategy (chooseSubGoal()). Indeed, when subgoals are defined, in order to have simpler sub-problems to solve, we only consider the first obstacle on the way and focus on getting pass this one. That relative myopia can yield to suboptimal decisions. The other main source of suboptimality is the use of history () to choose a subgoal. However, this provides consistency to the choosing strategy of directPath. Consistency is commonly used in wall-following based approaches [15] [16] and is known to be a good assurance for completeness (which is the property of solving any feasible problem). It has been proven for similar frameworks [17] [18]. Completeness of directPath remains to be analytically proven. However, during the heavy benchmarking we performed, it always succeeded in complete the planning task, which makes us confident about directPath completeness.

Finally, in Section V, we suggested potential benefits of combining directPath with other frameworks. More generally, it might be worth considering to couple directPath with any framework specially aimed to produce kinematic constrained paths [19] [20].

\section{REFERENCES}

[1] J.-C. Latombe, ROBOT MOTION PLANNING. Springer, 1990.

[2] T. Bailey and H. Durrant-Whyte, "Simultaneous localization and mapping (slam): Part ii," IEEE Robotics \& Automation Magazine, vol. 13, no. 3, pp. 108-117, 2006.

[3] M. Wang, W. Wang, J. Xiong, and L. Yan, "A consistent map building method based on surf loop closure detection," in Cyber Technology in Automation, Control and Intelligent Systems (CYBER), 2013 IEEE 3rd Annual International Conference on. IEEE, 2013, pp. 92-95.

[4] J. Mullane, B.-N. Vo, M. D. Adams, and B.-T. Vo, "A random-finite-set approach to bayesian slam," Robotics, IEEE Transactions on, vol. 27, no. 2, pp. 268-282, 2011

[5] S. S. Ge and Y. Cui, "Dynamic motion planning for mobile robots using potential field method," Autonomous Robots, vol. 13, 2002.

[6] Y. Koren and J. Borenstein, "Potential field methods and their inherent limitations for mobile robot navigation," in Robotics and Automation, 1991. Proceedings., 1991 IEEE International Conference on. IEEE, 1991, pp. 1398-1404.

[7] R. C. Arkin, "Motor schema-based mobile robot navigation," The International journal of robotics research, vol. 8, 1989.

[8] R. Brooks, "A robust layered control system for a mobile robot," Robotics and Automation, IEEE Journal of, vol. 2, no. 1, pp. 14-23, 1986.

[9] H. R. Beom and H. S. Cho, "A sensor-based navigation for a mobile robot using fuzzy logic and reinforcement learning," Systems, Man and Cybernetics, IEEE Transactions on, vol. 25, no. 3, pp. 464-477, 1995.

[10] Y. Gong, Y. Liu, and Z. Tang, "Path tracking of unmanned vehicle based on parameters self-tuning fuzzy control," in Cyber Technology in Automation, Control and Intelligent Systems (CYBER), 2013 IEEE 3rd Annual International Conference on. IEEE, 2013, pp. 52-57.

[11] Z. Fang and L. Zhang, "A multi-objective strategy based on frontierbased approach and fisher information matrix for autonomous exploration," in Cyber Technology in Automation, Control and Intelligent Systems (CYBER), 2013 IEEE 3rd Annual International Conference on. IEEE, 2013, pp. 96-101.

[12] P. E. Hart, N. J. Nilsson, and B. Raphael, "A formal basis for the heuristic determination of minimum cost paths," Systems Science and Cybernetics, IEEE Transactions on, vol. 4, no. 2, pp. 100-107, 1968.

[13] B. Todor and K. Csorba, "A method for autonomous robot navigation in partly known structured environments," in Intelligent Systems, 2004. Proceedings. 2004 2nd International IEEE Conference, vol. 3. IEEE, 2004, pp. 107-112.

[14] N. Sturtevant, "Benchmarks for grid-based pathfinding," Transactions on Computational Intelligence and AI in Games, vol. 4, no. 2, pp. 144 - 148, 2012. [Online]. Available: http://web.cs.du.edu/ sturtevant/ papers/benchmarks.pdf

[15] M. Katsev, A. Yershova, B. Tovar, R. Ghrist, and S. M. LaValle, "Mapping and pursuit-evasion strategies for a simple wall-following robot," Robotics, IEEE Transactions on, vol. 27, no. 1, pp. 113-128, 2011.

[16] X. Yun and K.-C. Tan, "A wall-following method for escaping local minima in potential field based motion planning," in Advanced Robotics, 1997. ICAR'97. Proceedings., 8th International Conference on. IEEE, 1997, pp. 421-426.

[17] I. Kamon and E. Rivlin, "Sensory-based motion planning with global proofs," Robotics and Automation, IEEE Transactions on, vol. 13, no. 6 , pp. 814-822, 1997.

[18] S. S. Ge, X. Lai, and A. A. Mamun, "Boundary following and globally convergent path planning using instant goals," Systems, Man, and Cybernetics, Part B: Cybernetics, IEEE Transactions on, vol. 35, no. 2, pp. 240-254, 2005.

[19] W. Song and Y. Hong, "Distributed relative attitude formation control of multi-agent systems with directed topology," in Cyber Technology in Automation, Control and Intelligent Systems (CYBER), 2013 IEEE 3rd Annual International Conference on. IEEE, 2013, pp. 1-6.

[20] Y. Kuroda and T. Saitoh, "Simultaneous adaptive path planning system for the real world application," in Robotic Intelligence in Informationally Structured Space, 2009. RIISS'09. IEEE Workshop on. IEEE, 2009, pp. $46-53$. 